\title{
ENGINEERING SUCCESS: USING PROBLEM-BASED LEARNING TO DEVELOP CRITICAL THINKING AND COMMUNICATION SKILLS
}

\author{
Jennifer L. Farmer ${ }^{1}$, Lydia Wilkinson ${ }^{2}$ \\ ${ }^{1}$ Department of Chemical Engineering and Applied Chemistry, ${ }^{2}$ Engineering Communication Program \\ University of Toronto \\ jennifer.farmer@utoronto.ca, lydia.wilkinson@utoronto.ca
}

\begin{abstract}
This paper discusses the implementation of a joint, end-of-term PBL exercise (a project lab) into our second-year chemical engineering curriculum at University of Toronto through two courses, Applied Chemistry Laboratory I (CHE204) and Communication (CHE299). The activity was designed to help students learn (i) research skills, (ii) how to select appropriate experiment techniques and equipment, (iii) how to design and conduct a research experiment, (iv) how to analyze real-world results, (v) how to communicate using a technical voice, (vi) how to work collaboratively in a group. Students were guided through the project with $e$ Learning modules, in-class active learning exercises, and written feedback, but were not provided with the type of detailed guidance typically provided in their LBL. Student feedback indicates that they are able to apply their learning from the activity to new contexts in a later assignment.
\end{abstract}

Keywords: Lab-based learning, problem-based learning (PBL), critical thinking, communication, chemical engineering, assignment design

\section{INTRODUCTION}

Engineers are problem-solvers, designers and builders. In order to create effective solutions to some of the most complex problems, engineers must have a working knowledge of nature and science that goes beyond a simple understanding of the theories and principles governing them: they must understand how these concepts are applied in practice. As a result, instructional laboratories, or LabBased Learning (LBL), has been an essential part of many undergraduate engineering programs, providing students with the opportunity to critically think about, discuss, and solve real-world problems in a safe learning environment $[1,2]$. While LBL is effective in promoting studentcentered, active learning pedagogies, conventional prescriptive approaches often used in undergraduate laboratories allow students to treat experimental procedures like recipes [3]. Students are less focused on thinking about why they are performing a specific experiment and more on how to follow the experimental procedure provided in the lab manual to obtain a correct result. While "recipe-driven" LBL experiments have their place in the teaching and learning of science, for example allowing students the opportunity to employ and verify fundamental theories taught in core courses, they usually do not provide students the opportunity to analyze the relevance of a lab and its application. As a result, students miss out on a crucial opportunity to engage in metacognition and improve retention, by understanding why they are learning what they are learning [4-9].

Problem-Based Learning (PBL) has been shown to promote these higher-level reasoning skills leading to better retention of knowledge. Similar to LBL, PBL is a student-centered teaching and learning approach in which students are challenged to think critically and analyze complex, real-world problems, with the problem as the vehicle for learning new knowledge [10]. Students are not presented directly with facts and concepts; instead, they must actively engage with the material through research, while integrating and organizing learned information and previous knowledge in new contexts [11]. Thus, the responsibility for learning has been shifted from the facilitator to the student, allowing the student to become a producer of knowledge rather than a consumer.

Despite the benefits of PBL pedagogy, its implementation into undergraduate chemical engineering laboratories, specifically ones focused on delivering chemistry content, has been limited, [3, 12-16]. Hesser and Bunyea describe the development and implementation of general chemistry PBL experiments (PBLE) to engineering students [16]. Cline and Powers describe the implementation of PBLE with unique open-ended design and research problems drawn from local industries [17]. In general, PBLE are introduced to students in upper year courses, once they have had an opportunity to learn fundamental concepts and laboratory techniques. Industry demands that students are able to apply fundamentals to a variety of problems. As educators, we want students to move from fundamental understanding to problem solving and application. Thus, we need to develop experiments that allow students to develop critical thinking and problem solving skills while reinforcing fundamentals. Furthermore, we need to expose students to this early in 
their studies. PBL was selected as an appropriate method to achieve these goals.

PBL also offers a valuable opportunity for students to develop and practice fundamental skills from other areas of the curriculum, notably research, teamwork and project management as they organize their approach to the project, and technical communication skills as they structure and write the report. As an exercise that bridges these interrelated skill groups it is also a vehicle to foster selfdirected, reflective, lifelong learners who can integrate knowledge, think critically, and work collaboratively with others, which are all desirable skills in the workplace.

While integrating a PBL project into the curriculum then, it was important to us that students learn (i) research skills, (ii) how to select appropriate experiment techniques and equipment, (iii) how to design and conduct a research experiment, (iv) how to analyze real-world results, (v) how to communicate using a technical voice, (vi) how to work collaboratively in a group. This paper discusses how we aimed to achieve these learning outcomes by implementing a joint PBL exercise (a project lab) through two courses, Applied Chemistry Laboratory I (CHE204) and Communication (CHE299) in the $2^{\text {nd }}$ year Chemical Engineering curriculum at University of Toronto.

\subsection{Context}

Currently, the Department of Chemical Engineering and Applied Chemistry offers two second-year laboratory courses (CHE204 - Applied Chemistry Laboratories I and CHE205 - Applied Chemistry Laboratories II). Both courses focus on applied chemistry laboratory techniques in the area of analytical, inorganic and organic chemistry. It is meant to supplement the learning experiences of the lectures in Applied Chemistry I (CHE220) and Applied Chemistry II (CHE213) from a practical point of view. Both courses are analytical and synthetic in nature. Students investigate chemical phenomena by probing the nature of the chemical samples provided to them, as well as synthesize inorganic and organic chemicals using established processes and laboratory techniques. Elements of process and industrial chemistry and practice (including Green Chemistry) are also explored.

CHE299: Communication is a standalone, full year communication course in the second year. The course supports the development of written, oral and visual communication skills across the curriculum. Students learn to_effectively identify and analyze audience and purpose, devise communication strategies to fulfill these goals, and present technical information persuasively and clearly for these different audiences while developing confidence as communicators. Course concepts are developed through active learning tutorials, where students engage in activities, small group and class discussions, selfassessment and peer review. While this course was originally devised to be closely integrated with core courses across the curriculum, in recent years content has shifted towards less integrated activities, often focused on professional and job search documents, which help students to situate themselves as future professionals, but are less immediately applicable to their educational success. This joint assignment provided an opportunity to reintroduce the type of cross-curricular activities that allow students to see the direct application of communication skills in engineering contexts.

\section{METHODS}

This year's project asked students to analyze two copper samples from two different mine sites to make a recommendation based not only on copper yield but additional factors such as social and environmental impact as well as long term economics. Students were required to research and justify the additional factors for consideration, and to integrate this research into their final recommendation. In preparation for the lab they were required to work collaboratively with a partner to develop their own protocol using theory and techniques taught in CHE204. Students received guidance on the project through in-class activities and online modules in both their CHE204 lab course and their CHE299 communication course.

The project was first shared with students on the course portal for CHE204, before being discussed in the CHE299 tutorial. In this course, it provided a basis for instructing students on research skills and library resources, and students were provided with an online lecture on keyword searches and using library databases as they wrote their introduction. This introduction, which required students to research their mine site and select relevant additional factors independently of their sample analysis, was graded in CHE299 approximately six weeks before the complete report was submitted for CHE204. The feedback they received on this early deliverable could be applied to strengthen research credibility and communication in their final report. Next, students were required to select their protocol in order to present it in a visual abstract for CHE299 in late October. This visual abstract assignment provided a vehicle for teaching visual design in the communication course, and was supported through multiple classes on diagram design, figure captioning and visual rhetoric; it also ensured that students were researching and selecting their visual protocol well in advance of their project lab due date. Both the introduction and visual abstract received peer review during tutorials before submission. Before students submitted their final experimental protocol they had an opportunity to bring their protocol to lecture for both peer and instructor review. Finally, the complete project lab document received an inclass peer review in CHE299, in advance of the final due 
date This sequence of deliverables is provided in Table 1 below.

Table 1. Sequence of deliverables

\begin{tabular}{|c|c|c|c|}
\hline Date & Course & Deliverable & Weight \\
\hline Oct 8 & CHE299 & $\begin{array}{l}\text { Introduction for } \\
\text { peer review }\end{array}$ & \\
\hline Oct 13 & CHE299 & Introduction due & $10 \%$ \\
\hline Oct 30 & CHE299 & $\begin{array}{l}\text { Visual abstract } \\
\text { for review }\end{array}$ & \\
\hline Nov $1 / 2$ & CHE204 & $\begin{array}{l}\text { Protocol for peer } \\
\text { review }\end{array}$ & \\
\hline Nov 3 & CHE299 & $\begin{array}{l}\text { Visual abstract } \\
\text { due }\end{array}$ & $10 \%$ \\
\hline Nov $8 / 9$ & CHE204 & Protocol due & $5 \%$ \\
\hline Dec 1 & $\begin{array}{l}\text { CHE204/ } \\
\text { CHE299 }\end{array}$ & $\begin{array}{l}\text { Final proposal } \\
\text { due }\end{array}$ & $\begin{array}{l}10 \%(299) \\
7 \%(204)\end{array}$ \\
\hline
\end{tabular}

Through this approach then, students were encouraged to take responsibility for their own learning at all stages of the project, while receiving frequent feedback from instructional teams in the two courses. The integrative nature of the project ensured that learning outcomes were achieved across the curriculum, as students practiced problem analysis, independent research, lab skills, persuasive writing and visual design.

\section{RESULTS AND DISCUSSION}

The success of this PBL activity was assessed in two ways. First, student grades on the CHE204 project lab were compared to grades on a similar project lab in their second semester CHE205 course; students were expected to complete this second lab with less in-class scaffolding, and as such it provided a measure of their success in retaining and applying the skills developed in the CHE204/299 assignment. Second, an online survey was distributed to gather student responses to the experience.

\subsection{Student Performance}

Despite the preparation provided through the CHE204 project lab, the class average did not improve between the two project iterations, and in fact decreased by $5.8 \%$ between the two courses, from $78.9 \%$ in CHE204 to $73.1 \%$ in CHE205. While this would seem to suggest a poorer overall performance, differences in the nature of the projects may account for the overall weaker average. Specifically, CHE204 is more focused on quantitative analysis, whereas CHE205 is more focused on qualitative analysis and identifying trends in data. This change in focus left many students wrestling with how to analyze and explain their data in the results and discussion section. Also, the mine sites and mining companies used in the
CHE204 assignment were selected from real-world examples, whereas those used in the CHE205 assignment was fictional. While reviewing students' introduction sections for the CHE205 assignment, it became evident that many saw this as a hurdle because they were unsure what to research and/or how to introduce this next project.

What's more, while the rubrics between the two assignments used the same assessment categories, grading was handled differently between the two courses. For the CHE204 project lab, the course instructor took responsibility for grading the abstract, methods, results, discussion and conclusions sections, and a communication instructor assessed overall coherence, and visual and written communication. Based on this grading distribution, students received a grade out of seven on technical content in CHE204, and a grade out of ten on communication in CHE299. (These two grades were aggregated to find overall performance grades for the purposes of this study.) For the CHE205 project labs, grades were determined using the same criteria, but sections and responsibilities were not split between graders or classes - the overall project was assessed as a component of the CHE205 class. With the exception of thirty reports co-assessed by the paper authors, complete project lab reports were graded by Chemical Engineering TAs, following a brief training and benchmarking session.

\subsection{Student Responses}

An end-of-year survey asked students to identify skills developed in the overall project lab and protocol component, to comment on the application of these skills in future, to describe changes in approach between CHE204 and CHE205, to comment on opportunities for reflection and peer feedback, and to provide recommendations for improvement in the CHE204/205 sequence. Distributing and allotting time to complete the online survey during CHE299 class time resulted in a fairly high completion rate, with 79 of 100 students completing at least one of the survey questions.

Responses varied considerably in all survey questions. Many students indicated that they developed visual communication skills, research, written communication and teamwork skills, with fewer students identifying time management skills and significantly less identifying oral communication and creativity outcomes. Responses to the question: "Please check the skills you developed through the overall project lab experiences" are summarized below. 




Fig. 1. Skills developed through the project labs

Students commented on the application of these skills in various contexts, and some moved beyond more obvious applications, such as in future lab projects and developing lab protocols, to comment on the value of problem solving (four students) and communication within a future professional environment (ten students). Students' comments on future applications have been sorted into common categories in Table 2 below.

Table 2. Future application of project lab skills

\begin{tabular}{|l|l|}
\hline Future Applications & 16 \\
\hline Lab assignments/reports & 15 \\
\hline Research projects & 10 \\
\hline Professional activities/workplace documents & 7 \\
\hline Protocols & 7 \\
\hline Managing time & 5 \\
\hline General communication activities & 5 \\
\hline Building visuals & 4 \\
\hline Working in teams & 4 \\
\hline Problem solving & 3 \\
\hline Delivering presentations & 12 \\
\hline N/A & \\
\hline
\end{tabular}

The range of activities identified here and their sometimesloose connection to the project content-as seen, for example, in the development of communication skillsmay be a product of the survey delivery. Because we presented the survey in CHE299 course time, some students appear to have considered all communication activities in their responses, rather than focusing on those that bridged the courses. Given that the second semester of the Communication course focuses on presentation skills, it is unsurprising that some students chose to acknowledge their improvement and continued application in these areas. Many responses however, spoke directly to the work done in this project sequence; one noted, for example: "Being able to solve problems in a practical setting is a very valuable experience. The team skills I gained, research abilities, and problem solving skills will be useful for my professional career. I should be able to think my way through any problem I have to solve" [18].
Targeted questions on the value of reflection in these projects provided us with feedback on how students engaged with and used the opportunities for reflection and improvement between the CHE204 and CHE205 projects. While some students valued these opportunities for reflection, noting that "the feedback from TAs and the graded rubrics were very helpful for my improvement," other students felt that there was insufficient reflection in the course. The perceived value of reflection activities is captured in Figure 2.



Fig. 2. Perceived value of reflection, with 5 being very valuable and 1 being not useful

Interestingly, while students were divided on the usefulness of reflection in the course, they overwhelmingly articulated one or more changes in their approach between the project labs for CHE204 and CHE205. In response to the prompt: "How did you change your approach to planning and writing the project lab between 204 and 205?" only twelve of the fifty-six respondents reported that they maintained the same approach. In fact, many shared specific strategies and improvements made. Student responses included:

- [I] budgeted more time [19].

- I tried to make the reasoning behind my protocol steps more explicit and clear [20].

- I decided to choose a new methodology when writing reports. I master the material first, understanding the theory. Then I examine the objectives of the assignment and brainstorm how I will integrate my current knowledge of principles to the objectives [21].

- For 205, I started a lot earlier giving me time to iterate over the course of two weeks, allowing me to produce the best document possible which accounts for all necessary information [22].

- I made sure I had effective research to support my argument. I made sure all the results were clearly organized in an excel sheet for clear communication. This helped me [to] clearly see 
the trends and [to] write the report with supporting evidence [23].

- I now start off before I begin writing [by] creating a plan. Before going into this course, I found [these] useless, but now creating an idea map can be extremely useful in guiding you through an assignment. Additionally, it makes writing the actual report a lot quicker [24].

The disconnect between the value placed on reflection, and apparent improvement in response to reflection may be due to the design of the activities meant to develop this practice. In one such activity, we asked students to review their graded CHE204 project lab, to identify specific areas for improvement, and to work with a partner to generate strategies to resolve these problems in their CHE205 project lab. This structured reflection activity was titled assignment review and goal-setting, rather than the more panic-inducing reflection, and as such students may have had trouble linking it to the survey prompt. Regardless of terminology, this activity was seemingly successful in helping students to implement changes between the first and second assignment through the type of meaningful metacognitive activity that we hoped would lead to improvement.

\section{CONCLUSION AND FUTURE DIRECTION}

The implementation of this joint PBL exercise was fairly successful in its first year. As we hoped, integration across the two courses provided opportunity to support student success in the project in various ways, through inclass planning and reflection activities, online modules and peer review. These activities effectively provided some direction, while still giving students the space to develop skills that will help them learn independently, namely critical thinking, problem solving and project management skills.

As noted above, student survey responses were somewhat inconsistent, with students simultaneously describing insufficient opportunity for reflection and articulating specific, actionable strategies for improvement. Given that we saw planned reflection as a means to ensure that students developed and retained learnt skills from one project to the next, sharing these specific strategies suggests success in this area.

Our metrics for success-student grades and the survey, provided less information on overall student satisfaction, but based on informal discussions with students over the year, we have generated a few areas for future improvement. One common complaint from students was a lack of consistent expectations and messaging from the teaching teams across the two courses. Given that this assignment and grading materials were collaboratively devised this complaint is somewhat unfounded (and may reflect overall frustration with grading rather than shed light on a real issue), but nevertheless this concern may be alleviated next year through a joint class or online lecture to kick-off the project. Optically, this team-taught class would emphasize the collaborative nature of the assignment planning.

In order to obtain more robust data next year, we will work to achieve greater consistency in grading between CHE204 and CHE205. Not only will we revisit the attribution of grades between the lab courses and CHE299, but we will also consider hiring graders that can assess these assignments across the full year.

With these changes we hope to more successfully meet the original aims of this assignment-researching and developing lab-based approaches to real world problems and developing the ability to effectively communicate their solution-while also refining how we measure our success in this area.

\section{References}

1. C. Davies Learning and Teaching in Laboratories, Higher Education Academy Engineering Subject Centre. 2008, [online] Available:

https://www.heacademy.ac.uk/system/files/learni ng-teaching-labs.pdf.

2. L. D. Feisel, A. J. Rose. "The Role of the Laboratory in Undergraduate Engineering Education". Engineering Education, vol. 94, no. 1, pp. 121-130, 2005.

3. O. C. Kelly, O. E. Finlauson. "Providing solutions through problem-based learning for the undergraduate $1^{\text {st }}$ year chemistry laboratory". Chemistry Education Research and Practice, vol. 8, no. 3, 347-361, 2007.

4. H. Vos, E. de Graaff. "Developing metacognition: A basis for active learning". European Journal of Engineering Education, vol. 29, no. 4, 543-548, 2004.

5. J. H. Flavel. "Metacognitive aspects of problem solving' in The nature of intelligence. L. Risnick, Ed. Lawrence Erlbaum Associates, Hillsdale, N.J., 1976.

6. C. Hmelo-Silver. "Problem-based learning: What and how do students learn?" Educational Psychology Review, vol. 16, no. 3, 235-266, 2004.

7. R. Sperling, B. Howard, R. Staley, N. Dubois. "Metacognition and self-regulated learning constructs". Educational Research and Evaluation: An International Journal on Theory and Practice, vo. 10, no. 2, 117-139, 2004. 
8. R. Marra, D.H. Jonassen, B. Palmer, S. Luft. "Why problem-based learning works: Theoretical foundations". Journal on Excellence in College Teaching, vo. 25, nos. 3\&4, 221-238, 2014.

9. Nor H. Ibrahim, Johari Surif, Khew P. Hui, Safiah Yaakub "“Typical" Teaching Method Applied in Chemistry Experiment Social and Behavioral Sciences, vol. 116, 4946 - 4954, 2014.

10. B. J. Duch, S. E. Groh, D. E. Allen. The Power of Problem-Based Learning, Sterling, VA: Stylus Publishing, 2001, pp. 274.

11. M. Z. Jamaludin, K. M. Yusof, N. F. Harun, S. A. H. S. Hassan. "Crafting Engineering Problems for Problem-Based Learning Curriculum". Procedia Social and Behavioural Sciences, vol. 56, pp. 377 $387,2012$.

12. R. Preetha. "Problem-Based Learning in Undergraduate Education - A Sophomore Chemistry Laboratory". Journal of Chemical Education, vol. 76, no. 8, 1122-1126, 1999.

13. C. McDonnell, C. O’Connor, M. K. Seery. "Developing Practical Chemistry Skills by Means of Student-Driven Problem-Based Learning Mini-Projects". Chemistry Education Research and Practice, vol. 8, no. 2, 130-139, 2007.

14. D. S. Domin. "A Review of Laboratory Instruction Styles". Journal of Chemical Education, vol. 76, no. 4, 543-547, 1999.

15. O. C. Kelly, O. E. Finlayson. "Providing Solutions Through Problem-Based Learning for the Undergraduate $1^{\text {st }}$ Year Chemistry Laboratory". Chemistry Education Research and Practice, vol. 8, no. 2, 347-361, 2007.
16. T. L. Hesser, and S. R. Bunyea (2016). "Development and Implementation of Problembased Chemistry Experiments for Engineering Students in a Multi-disciplinary Course." Paper presented at 2016 American Society for Engineering Education Annual Conference \& Exposition, New Orleans, Louisiana. https://peer.asee.org/27293

17. M. Cline, and G. J. Powers (1997). "Problem Based Learning via Open Ended Projects in Carnegie Mellon University's Chemical Engineering Undergraduate Laboratory" Paper presented at 1997 Frontiers in Education Conference $27^{\text {th }}$ Annual Conference, Pittsburgh, Pennsylvania, https://ieeexplore.ieee.org/document/644901/

18. Anonymous. Student Survey. CHE204/299 Integration, 2018.

19. Anonymous. Student Survey. CHE204/299 Integration, 2018.

20. Anonymous. Student Survey. CHE204/299 Integration, 2018.

21. Anonymous. Student Survey. CHE204/299 Integration, 2018.

22. Anonymous. Student Survey. CHE204/299 Integration, 2018.

23. Anonymous. Student Survey. CHE204/299 Integration, 2018.

24. Anonymous. Student Survey. CHE204/299 Integration, 2018. 